\title{
Vacinação anti-pneumocócica no idoso
}

Sofia Correia*

\section{RESUMO}

Objectivos: Determinar a efectividade e segurança da administração da vacina anti-pneumocócia polissacárida em idosos. Fontes de Dados: National Guideline Clearing House, Canadian Medical Association, Cochrane Library, TRIP database, Pubmed, Bandolier, Dare e Índex de Revistas Médicas Portuguesas.

Métodos de revisão: Pesquisa de normas de orientação clínicas, metanálises, revisões sistemáticas e ensaios clínicos aleatorizados e controlados publicados entre Maio de 2002 e Julho de 2012 nas línguas inglesa, portuguesa e espanhola. Foram utilizados os termos MeSH pneumococcal vaccines e aged. Na avaliação dos estudos e atribuição dos níveis de evidência e forças de recomendação foi utilizada a escala Strength of Recommendation Taxonomy (SORT) da American Family Physician.

Resultados: Foram encontrados 517 artigos. Destes, apenas 12 preencheram os critérios de inclusão, dos quais cinco normas de orientação clínica, três metanálises, três revisões sistemáticas e um ensaio clínico controlado e aleatorizado. Apesar da segurança da vacina, não existe consenso sobre a efectividade da mesma nos idosos. Os estudos observacionais revelaram uma efectividade de $55 \%$ a $59 \%$ na prevenção da doença pneumocócica invasiva, ao contrário dos ensaios clínicos aleatorizados e controlados incluídos nas metanálises e revisões sistemáticas avaliadas.

Conclusões: Perante a evidência disponível não foi possível determinar a efectividade da vacina anti-pneumocócica no idoso (Força de Recomendação B). São necessários ensaios clínicos controlados, aleatorizados, de elevada qualidade, de grandes dimensões, cuja amostra seja representativa da população de idosos para quem a vacina é recomendada, para obter conclusões firmes sobre a efectividade da mesma.

Palavras-chave: Vacina Anti-pneumocócica; Idoso.

\section{INTRODUÇÃO}

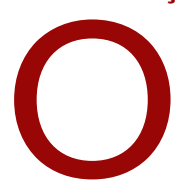

$\mathrm{s}$ idosos que adquirem infecções pneumocócicas são um grupo particularmente vulnerável a complicações como pneumonia, bacteriémia e meningite. Estas são, habitualmente, uma consequência da doença pneumocócica invasiva (DPI), que representa uma causa major de morbilidade em pessoas com 65 ou mais anos. ${ }^{1} \mathrm{Na}$ Europa a DPI atinge uma incidência de aproximadamente $45 \%$ neste grupo etário. ${ }^{2}$

Várias organizações, como o Centre for Disease Control and Prevention e The Royal Australian College of General Practitioners, recomendam a administração da vacina anti-pneumocócica por rotina nos idosos para prevenir estas patologias. Em Portugal, no entanto, não existem normas de orientação definidas sobre a prescrição da vacina neste grupo etário., ${ }^{3,4}$
A vacina pneumocócica polissacárida está incluída no Programa Nacional de Vacinação para administração a crianças e adolescentes que pertencem a grupos de risco (nomeadamente com antecedentes de drepanocitose e outras hemoglobinopatias, asplenia anatómica ou funcional, infecção pelo vírus da imunodeficiência humana, portadores ou candidatos a implante coclear, recém-nascidos prematuros e com síndrome de Down) e no Plano Regional de Vacinação da Região Autónoma dos Açores para administração a todas as crianças independentemente de pertencerem ou não a grupos de risco. O mesmo não acontece com os idosos, pelo que a prescrição desta vacina a todos os utentes com 65 ou mais anos de idade depende da recomendação do médico assistente e da sua noção sobre a efectividade desta medida preventiva. ${ }^{5,6}$

Na última década tem-se vindo a questionar a efectividade da vacina anti-pneumocócica na prevenção da pneumonia nos idosos, pelo que o objectivo deste 
estudo foi rever a evidência disponível sobre a efectividade e segurança da administração da vacina anti-pneumocócica polissacárida em idosos. ${ }^{7}$

\section{MÉTODOS}

Foi realizada uma pesquisa de normas de orientação clínicas (NOC), metanálises (MA), revisões sistemáticas (RS) e ensaios clínicos aleatorizados e controlados (ECAC) publicados nas bases de dados National Guideline Clearing House, Canadian Medical Association, Cochrane Library, TRIP database, Pubmed, Bandolier, Dare e Índex de Revistas Médicas Portuguesas entre Maio de 2002 e Julho de 2012 nas línguas inglesa, portuguesa e espanhola. Foram utilizados os termos MeSH pneumococcal vaccines e aged.

Foram incluídos na revisão artigos que cumprissem os seguintes critérios: a) População: idosos (pessoas com idade igual ou superior a 65 anos); b) Intervenção: vacina anti-pneumocócica polissacárida; c) Comparação: placebo ou ausência de vacinação; d) Outcome: efectividade (diminuição da incidência de doença pneumocócica invasiva, pneumonia pneumocócica ou pneumonia por todas as causas e diminuição da mortalidade) e incidência de efeitos adversos da vacina.

Foi utilizada a escala Strength of Recommendation Taxonomy (SORT) da American Family Physician na avaliação dos estudos e atribuição dos níveis de evidência e forças de recomendação.

\section{RESULTADOS}

Da pesquisa obtiveram-se 517 artigos. Foram excluídos os artigos que não cumpriam os objectivos ou os critérios de inclusão e os artigos repetidos. Dos artigos incluídos foram seleccionados: cinco NOC, três MA, três RS e um ECAC.

Na Austrália, a NOC do Royal Australian College of General Practitioners (Quadro I) recomenda a vacinação anti-pneumocócica em pessoas com idade igual ou superior a 65 anos, encontrando-se a vacina incluída no Plano Nacional de Vacinação australiano. Esta NOC menciona a efectividade da vacina na diminuição da incidência da DPI, mas não na pneumonia não-bacteriémica. ${ }^{4,8}$

A Public Health Agency of Canada aconselha a administração da vacina anti-pneumocócica a todos os idosos e considera a mesma eficaz na prevenção da DPI em $50 \%$ a $80 \%$ dos idosos. Refere que os efeitos adversos da vacina são ligeiros e manifestam-se como tumefacção, eritema ou febre ligeira. ${ }^{9}$

$\mathrm{O}$ Centres for Disease Control and Prevention recomenda, idealmente, a administração da vacina pneumocócica polissacárida com 23 serótipos a todas as pessoas com 65 anos, por existir evidência de que a efectividade da vacina diminui em pessoas com idade muito avançada. Aqueles que receberam a vacina antes dos 65 anos devem ser revacinados se já tiverem decorrido 5 anos desde a última dose. Esta NOC reconhece a eficácia e efectividade da vacina na prevenção da DPI, mas conclui existirem resultados contraditórios em relação à prevenção da pneumonia pneumocócica nãobacteriémica, definida como pneumonia sem isolamento de Streptococcus pneumoniae em locais habitualmente estéreis como sangue ou líquido cefalo-raquidiano. $^{3}$

O Department of Health (Reino Unido) aconselha a vacina pneumocócica polissacárida com 23 serótipos em toma única a todos os idosos. A vacina encontra-se incluída no plano de imunização do Reino Unido. Esta NOC refere a dificuldade em obter conclusões firmes sobre a efectividade da vacina, mas menciona a eficácia da mesma na prevenção da bacteriémia pneumocócica, aproximadamente 50 a $70 \% .^{10}$

A NOC da Infectious Diseases Society of America/American Thoracic Society, baseada na evidência e na opinião de peritos, recomenda a vacina pneumocócica polissacárida a todas as pessoas com idade superior a 65 anos na prevenção da DPI, considerando-a custo-eficaz. $^{11}$

Das três RS incluídas nesta revisão (Quadro II), a primeira foi efectuada por Watson e colaboradores em 2002 e analisou dezasseis ECAC cuja população era constituída por adultos e idosos, nos quais eram avaliados um ou mais dos seguintes outcomes: pneumonia pneumocócica, bacteriémia pneumocócica, pneumonia por todas as causas e mortalidade por todas as causas. Nos ECAC incluídos nesta RS nem todos os grupos de controlo receberam apenas placebo. Em três ECAC alguns elementos do grupo de controlo foram submetidos a vacina meningocócica, enquanto outros foram submetidos a placebo. Noutros dois ECAC o grupo de controlo recebeu apenas a vacina da gripe e em um ECAC foi administrada a vacina da gripe e placebo si- 


\begin{tabular}{|c|c|c|}
\hline Referência & Recomendação & $\begin{array}{c}\text { Força de } \\
\text { Recomendação }\end{array}$ \\
\hline $\begin{array}{l}\text { The Royal Australian College of } \\
\text { General Practitioners, } 2009^{4,8}\end{array}$ & $\begin{array}{l}\text { Todas as pessoas com } 65 \text { ou mais anos devem ser vacinadas com a } \\
\text { vacina pneumocócica polissacárida com } 23 \text { serótipos. }\end{array}$ & A \\
\hline Public Health Agency of Canada, $2006^{9}$ & $\begin{array}{l}\text { Todas as pessoas com } 65 \text { ou mais anos devem receber a vacina } \\
\text { anti-pneumocócica. }\end{array}$ & B \\
\hline $\begin{array}{l}\text { Centres for Disease Control and } \\
\text { Prevention, } 2010^{3}\end{array}$ & $\begin{array}{l}\text { A vacina pneumocócica polissacárida com } 23 \text { serótipos deve ser } \\
\text { administrada a todas as pessoas com } 65 \text { ou mais anos. }\end{array}$ & B \\
\hline Department of Health, $2011^{10}$ & $\begin{array}{l}\text { Por rotina deve ser oferecida uma dose única de vacina } \\
\text { anti-pneumocócica a todas as pessoas com } 65 \text { ou mais anos, se não } \\
\text { foi previamente administrada. }\end{array}$ & B \\
\hline $\begin{array}{l}\text { Infectious Diseases Society of America/ } \\
\text { /American Thoracic Society, } 2007^{11}\end{array}$ & $\begin{array}{l}\text { Todas as pessoas com idade superior a } 65 \text { anos devem receber a } \\
\text { vacina pneumocócica polissacárida para prevenir a DPI. }\end{array}$ & C \\
\hline
\end{tabular}

Legenda: DPI - Doença Pneumocócica Invasiva

multaneamente. Esta RS demonstrou a eficácia da vacina na diminuição da mortalidade e pneumonia por todas as causas em países não-industrializados, onde os participantes nos ECAC eram mais jovens, mas não em países industrializados, nos quais as amostras eram constituídas maioritariamente por idosos. Nos países industrializados, em idosos e pessoas imunocomprometidas ou com doença orgânica crónica, observou-se uma diminuição no número de casos de bacteriémia. No entanto não foi possível retirar conclusões firmes sobre este outcome devido ao pequeno número de casos. Os autores desta RS concluíram que o benefício da vacina poderá depender das características do hospedeiro e das características epidemiológicas da infecção na população-alvo. ${ }^{12}$

Na RS de Conaty e colaboradores, a análise dos estudos observacionais revelou que a vacina é efectiva na proteção de DPI em adultos e no subgrupo de idosos e adultos com doenças crónicas. Apesar da qualidade dos estudos observacionais que contribuíram para esta conclusão ser apenas moderada, os resultados foram consistentes e homogéneos. A efectividade da vacina na prevenção da pneumonia não foi demonstrada pelos poucos estudos observacionais heterogéneos que avaliavam este outcome. Os autores compararam estes resultados com aqueles obtidos na RS de ECAC publicada por Watson e colaboradores em 2002 e observaram que os resultados dos estudos observacionais eram consistentes com os dos ECAC relativamente à eficácia da vacina contra a DPI, mas não em relação à prevenção de pneumonia. ${ }^{13}$

A terceira RS analisada foi publicada por Loeb e colaboradores em 2004. Não apresenta protocolo explícito e critérios de inclusão e exclusão bem definidos, o processo de selecção, extracção e exclusão dos estudos não é detalhado e os autores não descrevem os métodos utilizados para avaliar o risco de viés, pelo que se considerou a qualidade desta RS como sendo pouco satisfatória. Os autores desta publicação analisaram cinco RS de ECAC e doze estudos observacionais. Os ECAC incluídos em quatro das cinco RS analisadas por Loeb e colaboradores não demonstraram benefício significativo da vacina relativamente à prevenção de pneumonia pneumocócica, pneumonia por todas as causas, bacteriémia e diminuição da mortalidade por pneumonia nos idosos. Apenas uma RS de ECAC revelou eficácia da vacina na prevenção de pneumonia pneumocócica nos idosos. Dos doze estudos observacionais incluídos na RS de Loeb e colaboradores, onze avaliavam os efeitos da vacina nos idosos, sendo que quatro demonstraram a efectividade da vacina na diminuição da incidência de bacteriémia, quatro na diminuição de DPI e um na redução do número de hospitalizações por pneumonia por todas as causas no gru- 


\begin{tabular}{|c|c|c|c|c|c|}
\hline Referência & $\begin{array}{l}\text { N. }{ }^{\circ} \text { de Estudos/ } \\
\text { /Outcomes Avaliados }\end{array}$ & Intervenção & Resultados & Comentários & NE \\
\hline $\begin{array}{l}\text { Watson et } \\
\text { al, 2002 }\end{array}$ & $\begin{array}{l}16 \text { ECAC } \\
\text { Outcomes avaliados: } \\
\text { pneumonia pneumocó- } \\
\text { cica, bacteriémia pneu- } \\
\text { mocócica, pneumonia } \\
\text { por todas as causas e } \\
\text { mortalidade por todas } \\
\text { as causas. }\end{array}$ & $\begin{array}{l}\text { Vacinação com VPP-6, } \\
\text { VPP-12, VPP-13 VPP-14, } \\
\text { VPP-17, VPP-23 vs } \\
\text { placebo, ou vacina da } \\
\text { gripe mais placebo ou } \\
\text { vacina meningocócica. } \\
\text { Países industrializados } \\
\text { vs países não-industria- } \\
\text { lizados. }\end{array}$ & $\begin{array}{l}\text { Em países industrializados } \\
\text { a vacina demonstrou } \\
\text { eficácia na diminuição } \\
\text { do número de casos de } \\
\text { bacteriémia } \\
\text { pneumocócica. } \\
\text { Em países não-industria- } \\
\text { lizados foi eficaz na } \\
\text { diminuição da } \\
\text { mortalidade e do } \\
\text { número de casos de } \\
\text { pneumonia por todas as } \\
\text { causas. }\end{array}$ & $\begin{array}{l}\text { Não determinaram a } \\
\text { segurança da vacina. } \\
\text { Concluíram que os } \\
\text { resultados díspares } \\
\text { podem dever-se às } \\
\text { características do } \\
\text { hospedeiro, agente e } \\
\text { meio ambiente. }\end{array}$ & 2 \\
\hline $\begin{array}{l}\text { Conaty et } \\
a l, 2004^{13}\end{array}$ & $\begin{array}{l}19 \text { ( } 11 \text { caso-controlo, } 8 \\
\text { coortes, } 2 \text { das quais } \\
\text { indirectas) } \\
\text { Outcomes avaliados: } \\
\text { doença pneumocócica } \\
\text { invasiva, pneumonia por } \\
\text { todas as causas. }\end{array}$ & $\begin{array}{l}\text { Vacinação com VPP-23 } \\
\text { ou VPP-14 vs ausência } \\
\text { de vacinação. }\end{array}$ & $\begin{array}{l}55 \% \text { de efectividade na } \\
\text { protecção contra doença } \\
\text { pneumocócica invasiva } \\
\text { nos idosos. } \\
\text { A vacina não se } \\
\text { demonstrou efectiva na } \\
\text { prevenção de pneumonia } \\
\text { por todas as causas. }\end{array}$ & $\begin{array}{l}\text { A estimativa da } \\
\text { efectividade da vacina } \\
\text { contra pneumonia por } \\
\text { todas as causas foi } \\
\text { baseada em alguns } \\
\text { estudos observacionais } \\
\text { heterogéneos. }\end{array}$ & 2 \\
\hline $\begin{array}{l}\text { Loeb et al, } \\
2004^{14}\end{array}$ & $\begin{array}{l}17 \text { ( } 5 \text { RS, } 5 \text { caso-controlo, } \\
3 \text { coortes retrospectivas, } \\
1 \text { coorte prospectiva, } \\
3 \text { coortes indirectas) } \\
\text { Outcomes avaliados: } \\
\text { pneumonia pneumo- } \\
\text { cócica, bacteriémia } \\
\text { pneumocócica, pneu- } \\
\text { monia por todas as } \\
\text { causas e mortalidade } \\
\text { por todas as causas. }\end{array}$ & $\begin{array}{l}\text { Vacinação com a VPP vs } \\
\text { ausência de vacinação }\end{array}$ & $\begin{array}{l}\text { A vacina anti-pneumo- } \\
\text { cócica polissacárida não } \\
\text { mostrou benefício } \\
\text { significativo nos ECAC } \\
\text { incluídos na revisão, ao } \\
\text { contrário dos estudos } \\
\text { observacionais. }\end{array}$ & $\begin{array}{l}\text { Não se observaram } \\
\text { efeitos adversos } \\
\text { significativos. }\end{array}$ & 2 \\
\hline
\end{tabular}

Legenda: NE - Nível de Evidência; ECAC - Ensaios Clínicos Aleatorizados e Controlados; VPP - Vacina Pneumocócica Polissacárida; RS - Revisões Sistemáticas.

po vacinado. Em relação aos efeitos adversos, esta RS descreve as reacções locais como dor no local da injecção, eritema e edema como os mais frequentes. Numa das RS de ECAC incluídas no trabalho de Loeb e cola- boradores, os efeitos adversos ocorreram em menos de um terço dos 7531 idosos vacinados, não tendo ocorrido nenhum caso de anafilaxia. ${ }^{14}$

Na primeira das três MA incluídas nesta revisão 


\begin{tabular}{|c|c|c|c|c|c|}
\hline Referência & $\begin{array}{c}\text { N. }{ }^{\circ} \text { de Estudos/ } \\
\text { /Outcomes Avaliados }\end{array}$ & Intervenção & Resultados & Comentários & NE \\
\hline $\begin{array}{l}\text { Puig- } \\
\text { Barbera et } \\
\text { al, } 2002^{15}\end{array}$ & $\begin{array}{l}20 \text { ( } 11 \text { ensaios clínicos, } \\
\text { dos quais apenas } 5 \text { eram } \\
\text { ECAC, } 9 \text { observacionais). } \\
\text { Outcomes avaliados: } \\
\text { pneumonia } \\
\text { pneumocócica, doença } \\
\text { pneumocócica invasiva. }\end{array}$ & $\begin{array}{l}\text { Vacinação com a VPP vs } \\
\text { placebo, vacina de } \\
\text { controlo ou ausência de } \\
\text { intervenção. }\end{array}$ & $\begin{array}{l}\text { O RR de pneumonia } \\
\text { pneumocócica dos } \\
\text { vacinados vs não- } \\
\text {-vacinados foi de } 0,86 \\
\text { (IC } 95 \%, 0,24 \text { a } 2,99 \text { ) e } \\
\text { a efectividade da vacina } \\
\text { foi de } 14 \% \text { (IC } 95 \% \text {, } \\
\text {-199 a } 76 \% \text { ). } \\
\text { O RR de DPI foi de 0,68 } \\
\text { (IC a } 95 \%, 0,39 \text { a 1,18) e } \\
\text { a efectividade da vacina } \\
\text { na protecção de DPI foi } \\
\text { de } 32 \% \text { (IC a } 95 \%,-18 \\
\text { a } 61 \% \text { ). }\end{array}$ & $\begin{array}{l}\text { O RR de pneumonia } \\
\text { pneumocócica foi } \\
\text { calculado com base num } \\
\text { ECAC. } \\
\text { O RR de DPI foi calculado } \\
\text { com base em } 2 \text { ECAC e } 2 \\
\text { estudos observacionais. }\end{array}$ & 2 \\
\hline $\begin{array}{l}\text { Moberley et } \\
\text { al, } 2008^{16}\end{array}$ & $\begin{array}{l}22 \text { (15 ECAC, } 5 \text { caso- } \\
\text {-controlo e } 2 \text { coortes) } \\
\text { Outcomes avaliados: } \\
\text { pneumonia pneumo- } \\
\text { cócica, doença pneumo- } \\
\text { cócica invasiva, mortali- } \\
\text { dade por infecção pneu- } \\
\text { mocócica, mortalidade } \\
\text { por pneumonia. }\end{array}$ & $\begin{array}{l}\text { Vacinação com a VPP-2, } \\
\text { VPP-3, VPP-6, VPP-12, } \\
\text { VPP-13, VPP-14, VPP-17, } \\
\text { VPP-23 ou VPP-14 e } \\
\text { vacina da gripe vs } \\
\text { placebo, vacina da gripe, } \\
\text { vacina meningocócica ou } \\
\text { ausência de intervenção. }\end{array}$ & $\begin{array}{l}\text { A vacina demonstrou } \\
\text { efectividade de } 59 \% \text { na } \\
\text { prevenção da DPI em } \\
\text { idosos imunocompe- } \\
\text { tentes, que não foi } \\
\text { estatisticamente } \\
\text { significativa. } \\
\text { A efectividade da vacina } \\
\text { na protecção de } \\
\text { pneumonia por todas as } \\
\text { causas e diminuição da } \\
\text { mortalidade por todas as } \\
\text { causas foi inconclusiva. }\end{array}$ & $\begin{array}{l}\text { Incluiu pessoas com } \\
\text { idade > } 16 \text { anos. Foram } \\
\text { efectuadas análises de } \\
\text { subgrupos para } \\
\text { determinar a eficácia da } \\
\text { vacina em diferentes } \\
\text { grupos. }\end{array}$ & 2 \\
\hline $\begin{array}{l}\text { Huss et al, } \\
2009^{17}\end{array}$ & $\begin{array}{l}22 \text { ECAC } \\
\text { Outcomes avaliados: } \\
\text { pneumonia pneumo- } \\
\text { cócica, bacteriémia } \\
\text { pneumocócica, bronquite, } \\
\text { pneumonia por todas as } \\
\text { causas, mortalidade por } \\
\text { todas as causas, mortali- } \\
\text { dade por infecção } \\
\text { pneumocócica, mortali- } \\
\text { dade por pneumonia. }\end{array}$ & $\begin{array}{l}\text { Vacinação com a VPP-3, } \\
\text { VPP-4, VPP-6, VPP-12, } \\
\text { VPP-13, VPP-14, VPP-17 } \\
\text { ou VPP-23 vs placebo, } \\
\text { vacina de controlo ou } \\
\text { ausência de intervenção. }\end{array}$ & $\begin{array}{l}\text { O RR de pneumonia } \\
\text { pneumocócica foi de } \\
1,04 \text { (IC a } 95 \%, 0,78 \text { a } \\
1,38 \text { ). } \\
\text { O RR de pneumonia por } \\
\text { todas as causas foi de } \\
0,89 \text { (IC a } 95 \%, 0,69 \text { a } \\
\text { 1,14). } \\
\text { O RR de mortalidade por } \\
\text { todas as causas foi de } \\
1,00 \text { (IC a } 95 \%, 0,87 \text { a } \\
1,14 \text { ). }\end{array}$ & $\begin{array}{l}\text { O RR de pneumonia } \\
\text { pneumocócica foi } \\
\text { calculado com base em } \\
7 \text { ECAC. } \\
\text { O RR de pneumonia por } \\
\text { todas as causas foi } \\
\text { calculado com base em } \\
11 \text { ECAC. } \\
\text { O RR de mortalidade por } \\
\text { todas as causas foi } \\
\text { calculado com base em } \\
10 \text { ECAC. }\end{array}$ & 2 \\
\hline
\end{tabular}

Legenda: NE - Nível de Evidência; ECAC - Ensaios Clínicos Aleatorizados e Controlados; VPP - Vacina Pneumocócica Polissacárida; RR - Risco Relativo; IC - Intervalo de Confiança; DPI - Doença Pneumocócica Invasiva 
(Quadro III), Puig-Barbera e colaboradores concluíram que a vacina anti-pneumocócica não é eficaz na diminuição do número de casos de doença por Streptococcus pneumoniae em pessoas com mais de 55 anos. No entanto, apenas cinco ECAC foram incluídos para avaliar este outcome, retirando poder à metanálise. Em alguns ECAC avaliados nesta MA, o grupo de controlo foi submetido a outras vacinas em vez de apenas placebo, mas os autores da MA não descrevem quais foram as vacinas utilizadas nesses estudos. ${ }^{15}$

Os ECAC incluídos na MA de boa qualidade metodológica da Cochrane, efectuada por Morbeley e colaboradores, demonstraram uma efectividade de $74 \%$ da vacina na prevenção da DPI em pessoas com idade superior a 16 anos. Ao analisar os resultados obtidos por subgrupos não foi comprovada a eficácia protectora da vacina na DPI em pessoas com mais de 55 anos e doen- ça crónica, provavelmente por falta de poder dos estudos e heterogeneidade dos mesmos. A vacina demonstrou, porém, uma efectividade de $59 \%$ em idosos imunocompetentes, que não foi estatisticamente significativa. Esta metanálise não avaliou os efeitos adversos da vacina. ${ }^{16}$

Na metanálise efectuada por Huss e colaboradores observou-se um elevado grau de heterogeneidade entre os estudos que avaliavam a eficácia da vacina para diferentes outcomes devido a diferenças metodológicas. A evidência foi insuficiente na demonstração dos efeitos preventivos da vacina na pneumonia pneumocócica, pneumonia por todas as causas, diminuição da mortalidade nos idosos ou adultos com doença respiratória crónica e na prevenção de DPI (RR combinado de 0,90, IC 95\% 0,46 - 1,77) em adultos e idosos. Dos estudos analisados, não foram registados efeitos adver-

\section{QUADRO IV. Ensaios Clínicos Aleatorizados e Controlados}

\begin{tabular}{|c|c|c|c|c|c|}
\hline Referência & População & Intervenção & Endpoint & Resultados & NE \\
\hline $\begin{array}{l}\text { Maruyama } \\
\text { et al } 2010^{18}\end{array}$ & $\begin{array}{l}\text { Residentes em } \\
\text { lares de idosos } \\
\text { no Japão com } \\
\text { idades entre os } \\
55 \text { e } 105 \text { anos } \\
n=1006 .\end{array}$ & $\begin{array}{l}\text { VPP-23 } \\
(n=502) \\
\text { vs } \\
\text { Placebo } \\
(n=504)\end{array}$ & $\begin{array}{l}\text { Primário: determinar a } \\
\text { incidência de pneumonia } \\
\text { pneumocócica e } \\
\text { pneumonia por todas as } \\
\text { causas. } \\
\text { Secundário: determinar o } \\
\text { número de mortes por } \\
\text { pneumonia pneumo- } \\
\text { cócica, pneumonia não- } \\
\text {-pneumocócica e } \\
\text { pneumonia por todas as } \\
\text { causas. }\end{array}$ & $\begin{array}{l}\text { Redução na incidência de pneumonia } \\
\text { pneumocócica: } 63,8 \% \text { (IC } 95 \%, 32,1 \% \text { a } \\
80,7 \% \text { ); p<0,05 } \\
\text { Redução na incidência de pneumonia } \\
\text { não-pneumocócica: } 29,4 \% \text { (IC } 95 \%,-4,3 \% \\
\text { a 52,3\%); p } \geq 0,05 \\
\text { Redução na incidência de pneumonia por } \\
\text { todas as causas: } 44,8 \% \text { (IC } 95 \%, 22,4 \% \text { a } \\
60,8 \% \text { ); p<0,05 } \\
\text { Mortalidade por pneumonia pneumocócica: } \\
\text { VPP-23 vs Placebo } 0 / 14 \text { (0\%) vs } 13 / 37 \\
\text { (35,1\%) p<0,05 } \\
\text { Mortalidade por pneumonia não- } \\
\text {-pneumocócica: VPP-23 vs Placebo 13/49 } \\
\text { (26,5\%) vs } 13 / 67 \text { ( } 19,4 \% \text { ) p } \geq 0,05 \\
\text { Mortalidade por pneumonia por todas as } \\
\text { causas: VPP-23 vs Placebo 13/63 ( } 20,6 \% \text { ) } \\
\text { vs } 26 / 104 \text { ( } 25,0 \% \text { ) p } \geq 0,05\end{array}$ & 1 \\
\hline
\end{tabular}


sos graves a curto prazo. Tal como na MA de Puig-Barberà e colaboradores, em alguns ECAC incluídos nesta MA o grupo de controlo foi submetido a outras vacinas, mas Huss e colaboradores não descrevem quais foram as vacinas utilizadas. ${ }^{17}$

O único ECAC incluído (Quadro IV) foi efectuado por Maruyama e colaboradores e publicado em 2010. Teve como principal objectivo avaliar a efectividade da vacina anti-pneumocócica polissacárida 23 -valente (VPP-23) na prevenção da pneumonia pneumocócica e pneumonia por todas as causas em residentes em lares de idosos. É um estudo de boa qualidade, com dupla-ocultação assegurada, boa dimensão amostral e um follow-up superior a 80\% (dos 1006 participantes, 837 concluíram o estudo). Em 502 dos 1006 participantes foi administrada aVPP-23, que se revelou eficaz na prevenção de pneumonia pneumocócica e pneumonia por todas as causas. A mortalidade por pneumonia pneumocócica foi significativamente superior no grupo que não recebeu a vacina anti-pneumocócica. Não houve diferença estatisticamente significativa relativamente a morte por pneumonia não-pneumocócica e pneumonia por todas as causas. ${ }^{18}$

\section{CONCLUSÕES}

Perante a evidência disponível, não foi possível determinar a efectividade da vacina na prevenção da DPI e na diminuição da mortalidade e do número de casos de pneumonia pneumocócica ou pneumonia por todas as causas (Força de Recomendação B).

De realçar que a falta de um método de diagnóstico específico limita a capacidade de detectar um efeito protector da vacina contra a pneumonia pneumocócica. A utilização de diferentes metodologias, a selecção de amostras constituídas por diferentes grupos etários que não representam a população em causa, a escolha de diferentes outcomes e a utilização de diferentes tipos de vacinas anti-pneumocócicas (conjugadas ou não conjugadas e com diferentes tipos de serótipos), contribuem para a heterogeneidade dos estudos até agora efectuados sobre a eficácia da vacina, diminuindo a firmeza das conclusões obtidas.

São necessários ECAC de elevada qualidade e de grandes dimensões, cuja amostra seja representativa da população de idosos para quem a vacina é recomendada e que seja submetida a um follow-up ade- quado para obter conclusões firmes sobre a efectividade da vacina.

\section{AGRADECIMENTOS}

À Orientadora de Formação da autora: Dra. Fátima Brandão.

\section{REFERÊNCIAS BIBLIOGRÁFICAS}

1. World Health Organisation. Pneumococcal vaccines: WHO position paper. Wkly Epidemiol Rec 1999 Jun 11; 74 (23): 177-83. Disponível em: http://www.who.int/docstore/wer/pdf/2003/wer7814.pdf [acedido em 06/05/2012].

2. European Centre for Disease Prevention and Control. Surveillance of invasive pneumococcal disease in Europe, 2010. Stockholm: ECDC; 2012. Disponível em: http://www.ecdc.europa.eu/en/publications/Publications/invasive-pneumoccocal-disease-surveillance-2010.pdf [acedido em 22/02/2013].

3. Centres for Disease Control and Prevention. Updated recommendations for prevention of invasive pneumococcal disease among adults using the 23-valent pneumococcal polysaccharide vaccine (PPSV23). MMWR Morb Mortal Wkly Rep 2010 Sep 3; 59 (34): 1102-6.

4. The Royal Australian College of General Practitioners. Guidelines for Preventive Activities in General Practice. 7th ed. Melbourne:The Royal Australian College of General Practitioners; 2009. p. 28.

5. Direcção-Geral da Saúde. Vacinação, a nível hospitalar, contra infecções por Streptococcus pneumoniae de crianças/adolescentes de risco para doença invasiva pneumocócica (DIP). Circular Normativa N¹2/DSPD, 09/06/2010. Lisboa: Ministério da Saúde; 2010.

6. Decreto Legislativo Regional. Portaria n. ${ }^{\circ}$ 104/2011, de 29 de Dezembro de 2011. Jornal Oficial da Presidência do Governo dos Açores - Série $\mathrm{I}, \mathrm{n}^{\circ} 186$.

7. Artz AS, Ershler WB, Longo DL. Pneumococcal vaccination and revaccination of older adults. Clin Microbiol Rev 2003 Apr; 16 (2): 308-18.

8. Australian Government Department of Health and Ageing. The Australian Immunization Handbook. 9th ed. Melbourne: Royal Australian College of General Practitioners; 2008. Disponível em: http://www.racgp.org.au/guidelines [acedido em 19/06/2012].

9. Public Health Agency of Canada. Canadian Immunization Guide. 7th ed. 2006. Disponível em: http://www.phac-aspc.gc.ca/publicat/ciggci/index-eng.php [acedido em 19/06/2012].

10. Department of Health. Immunization against infectious disease. 2nd ed. 2006. Disponível em: http://www.dh.gov.uk/prod_consum_dh/ groups/dh_digitalassets/@dh/@en/documents/digitalasset/dh_13311 8.pdf [acedido em 19/06/2012].

11. Mandell LA, Wunderink RG, Anzueto A, Bartlett JG, Campbell GD, Dean NC, et al. Infectious Diseases Society of America/American Thoracic Society consensus guidelines on the management of community-acquired pneumonia in adults. Clin Infect Dis 2007 Mar 1; 44 (2): S2772.

12. Watson L, Wilson BJ, Waugh N. Pneumococcal polysaccharide vaccine: a systematic review of clinical effectiveness in adults. Vaccine 2002 May 22; 20 (17-18): 2166-73.

13. Conaty S, Watson L, Dinnes J, Waugh N. The effectiveness of pneumococcal polysaccharide vaccines in adults: a systematic review of ob- 
servational studies and comparison with results from randomised controlled trials. Vaccine 2004 Aug 13; 22 (23-24): 3214-24.

14. Loeb M, Stevenson KB, SHEA Long-Term-Care Committee. Pneumococcal immunization in older adults: implications for the long-termcare setting. Infect Control Hosp Epidemiol 2004 Nov; 25 (11): $985-$ 94.

15. Puig-Barberà J, BelenguerVarea A, Goterris Pinto M, Brines Benlliure MJ. Efectividad de la vacuna frente al neumococo en el anciano: revisión sistematica y metaanalisis. Aten Primaria 2002 Sep 30; 30 (5): 269-81.

16. Moberley SA, Holden J, Tatham DP, Andrews RM. Vaccines for preventing pneumococcal infection in adults. Cochrane Database Syst Rev 2008 Jan 23; (1): CD000422.

17. Huss A, Scott P, Stuck AE, Trotter C, Egger M. Efficacy of pneumococcal vaccination in adults: a meta-analysis. CMAJ 2009 Jan 6; 180 (1): 48-58.

18. Maruyama T, Taguchi O, Niederman MS, Morser J, Kobayashi H,
Kobayashi T, et al. Efficacy of 23-valent pneumococcal vaccine in preventing pneumonia and improving survival in nursing home residents: double blind, randomised and placebo controlled trial. BMJ 2010 Mar 8; 340: 1004 .

\section{CONFLITOS DE INTERESSE}

A autora declara não ter conflito de interesses.

\author{
ENDEREÇO PARA CORRESPONDÊNCIA \\ Sofia Feijó Correia \\ Rua da Natividade $n^{\circ} 9$ \\ 9560-215 Lagoa \\ E-mail: sofia.fj.correia@gmail.com
}

Recebido em 16/10/2012

Aceite para publicação em 13/06/2013

\section{ABSTRACT}

\section{PNEUMOCOCCAL VACCINE IN THE ELDERLY: AN EVIDENCE BASED REVIEW}

Objectives: To determine the effectiveness and safety of pneumococcal vaccine in the elderly.

Data Sources: National Guideline Clearing House, Canadian Medical Association, Cochrane Library, TRIP database, Pubmed, Bandolier, Dare and Índex de Revistas Médicas Portuguesas.

Review methods: A search for clinical practice guidelines, systematic reviews, meta-analyses and randomized controlled trials published between May 2002 and July 2012 was performed using the MeSH terms "pneumococcal vaccines" and "aged". The Strength of Recommendation Taxonomy scale of the American Family Physician was applied to evaluate the quality of the studies and the level of evidence.

Results: We found 517 articles, but only twelve met the inclusion criteria. These included five clinical practice guidelines, three meta-analyses, three systematic reviews and one randomized controlled trial. Studies were inconsistent regarding the effectiveness of pneumococcal vaccine in the elderly, despite its safety. Observational studies showed that the vaccine has an effectiveness of $55 \%$ to $59 \%$ in preventing invasive pneumococcal disease, in contrast with the randomized controlled clinical trials included in the meta-analyses and systematic reviews evaluated which did not demonstrate benefit.

Conclusions: The available evidence is not strong enough to support the routine use of the pneumococcal vaccine in the elderly (Strength of Recommendation B). Additional high quality randomized controlled trials with a representative sample of the population for whom the vaccine is recommended are necessary to assess the need for the pneumococcal vaccine.

Keywords: Pneumococcal Vaccine; Aged. 